\title{
Absolute extinction cross-section of individual magnetic split-ring resonators
}

\author{
MARTIN HUSNIK ${ }^{1}$, MATTHIAS W. KLEIN ${ }^{1}$, NILS FETH ${ }^{1,2 *}$, MICHAEL KÖNIG ${ }^{3}$, JENS NIEGEMANN ${ }^{3}$, \\ KURT BUSCH ${ }^{2,3}$, STEFAN LINDEN ${ }^{1,2}$ AND MARTIN WEGENER ${ }^{1,2}$
}

${ }^{1}$ Institut für Angewandte Physik, Universität Karlsruhe (TH), Wolfgang-Gaede-Straße 1, D-76131 Karlsruhe, Germany ${ }^{2}$ Institut für Nanotechnologie, Forschungszentrum Karlsruhe in der Helmholtz-Gemeinschaft, D-76021 Karlsruhe, Germany ${ }^{3}$ Institut für Theoretische Festkörperphysik, Universität Karlsruhe (TH), Wolfgang-Gaede-Straße 1, D-76131 Karlsruhe, Germany *e-mail: nils.feth@physik.uni-karlsruhe.de

Complete control of an electromagnetic wave requires access to its electric and magnetic vector components. Realizing this level of control with metamaterials has recently opened new avenues regarding negative refractive indices ${ }^{1,2}$ and invisibility cloaking ${ }^{3,4}$. The required microscopic building blocks are artificial electric and magnetic dipoles. Magnetic dipoles oscillating at optical frequencies have become available only recently in the form of man-made split-ring resonators ${ }^{5}$, essentially subwavelength resonant electromagnets. Previous experimental work has focused on arrays of electric and/or magnetic dipoles ${ }^{1,2,6,7}$. For further developments in this field, knowledge of the properties of the individual dipoles is highly desirable. In this paper, using a modulation technique ${ }^{8,9}$, we measure the absolute extinction cross-section of a single splitring resonator for the first time. At the fundamental magnetic resonance, it is found to be about one-seventh of $\lambda^{2}$ at a wavelength of $\lambda=1.4 \mu \mathrm{m}$, which is in excellent agreement with microscopic calculations.

Metamaterials are man-made structures that are composed of densely packed subwavelength (metallic) building blocks ${ }^{1-7}$. Although the properties of the individual building blocks (photonic atoms) will mainly determine the optical properties of the metamaterials, interaction effects also play a role $e^{10-12}$. Thus, it is relevant to investigate the optical properties of the individual photonic atoms, that is, to measure their absolute extinction cross-section spectrum, a measurement not achievable using either dark-field spectroscopy ${ }^{13}$ or attenuated total internal reflection spectroscopy. This task has been accomplished only quite recently for electric dipole Mie resonances ${ }^{8,9}$. The absolute extinction cross-section spectrum for artificial magnetic dipoles, specifically split-ring resonators (SRRs), will be measured and theoretically analysed in this paper.

In geometrical optics, introducing an object with a crosssection $C_{\text {ext }}\left(C_{\text {ext }} \leq A\right)$ into a beam of light with area $A$ simply reduces the energy flow into the forward direction in the far-field by the fraction $C_{\text {ext }} / A \leq 1$. In wave optics and for a subwavelength scatterer in the focus of a Gaussian beam (Fig. 1), a detailed analysis based on the optical theorem ${ }^{14}$ shows that the energy flow into the forward direction is reduced by the same fraction $C_{\text {ext }} / A$ if $A$ is replaced by the effective beam area of the Gaussian focus. Typically, $A$ is significantly larger than $\lambda^{2}$, but even the resonant extinction cross-section is usually only a fraction of $\lambda^{2}$. Thus, a small focus, an intense broadband light source and a sensitive modulation technique enabling lock-in detection of the small relative signal $C_{\text {ext }}(\lambda) / A$ are required. Quantitative experimental knowledge of $A$ and the spatial modulation amplitude then allow us to infer the absolute extinction cross-section and its spectral dependence $C_{\text {ext }}(\lambda)$ directly from experiment (see Methods).

Examples of the resulting sets of raw data are shown in Fig. 2a. As expected from previous works ${ }^{8,9}$, depending on whether the modulation period starts or ends at the scatterer, a maximum or a minimum is obtained in the differential transmittance signal. For these data, the modulation amplitude has been fixed at $a=3 \mu \mathrm{m}$. The extinction cross-section $C_{\text {ext }}$ is given by $C_{\text {ext }}(\lambda)=A \times \mathrm{RDS}_{\max } / \xi . \mathrm{RDS}_{\max }$ is the maximum of the relative differential signal in the $x y$-plane (see Fig. 2a). The calibration factor $\xi$ is given by

$$
\xi=\max _{y}\left\{\sqrt{2 \pi} \int_{-\infty}^{+\infty} r_{0} \mathrm{e}^{2 \pi \mathrm{i} \kappa y} \mathrm{i} J_{1}(2 \pi \kappa a) \mathrm{e}^{-\pi^{2} r_{0}^{2} \kappa^{2} / 2} \mathrm{~d} \kappa\right\},
$$

where $J_{1}$ is the Bessel function of the first kind. The dependence $\xi\left(a / r_{0}\right)$ is illustrated in Fig. 1c.

For the horizontal incident linear polarization shown in Fig. 2b, the measured extinction cross-section of an individual SRR reveals a pronounced spectral maximum at a wavelength of $\sim 1.4 \mu \mathrm{m}$. Arrays of very similar SRRs have previously been investigated and this resonance has been ascribed to a magnetic dipole mode ${ }^{7}$. The spectral position is also in good agreement with complete numerical solutions of the vector Maxwell equations (see below). To further support this interpretation, we also depict the extinction crosssection spectrum for the orthogonal incident linear polarization of light in Fig. 2b. The resonance at $\sim 1.4 \mu \mathrm{m}$ disappears, as expected from the results obtained for arrays ${ }^{7}$. In essence, the incident electric field can no longer couple to the capacitor that is formed by the two vertical arms of the SRR. Together with the inductor provided by the incomplete single winding of a coil, this capacitor forms a resonant $L C$ circuit-a tiny electromagnet with a magnetic dipole moment perpendicular to its plane. This resonance is the fundamental plasmonic mode ${ }^{15}$ of the split ring.

Figure 2c summarizes results for a sample different from but very similar to that in Fig. 2b. The cross-section spectra in 


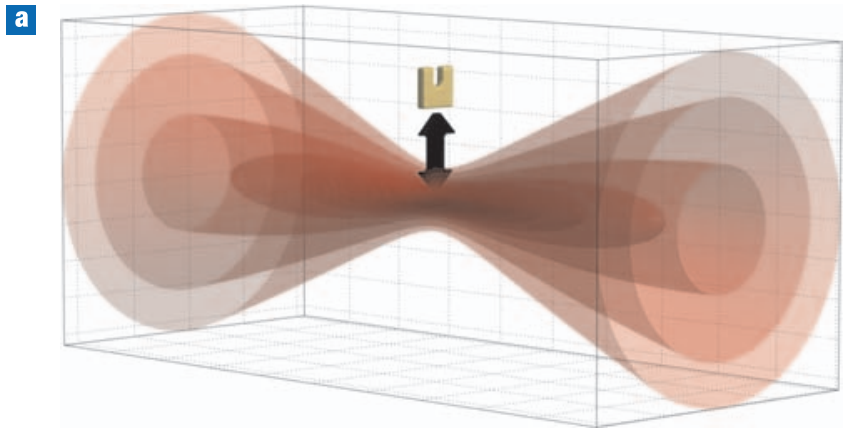

b

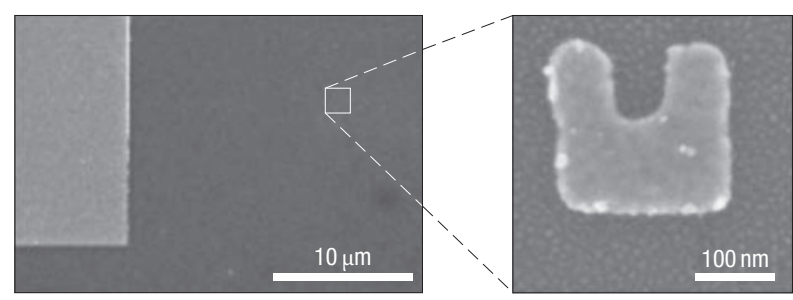

$\mathbf{6}$

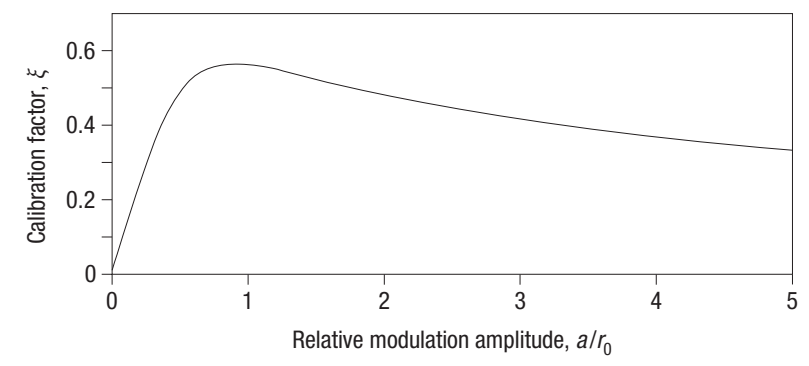

Figure 1 Determining the absolute extinction cross-section. a, Schematic of the modulation technique. The SRR is periodically modulated along the $y$-direction, in and out of the Gaussian focus, which is illustrated by different iso-intensity contours (red lobes). b. Sample layout and electron micrograph of a typical individual SRR. c, Calibration factor $\xi$ versus the ratio of the modulation amplitude $a$ and the Gaussian beam radius $r_{0}$. The extinction cross-section $C_{\text {ext }}$ is given by the product of the effective Gaussian beam area $A$ and the maximum of the relative differential signal RDS (also see Fig. 2a) obtained from a lock-in amplifier, divided by the calibration factor $\xi$.

Fig. 2b,c agree well with respect to their shape as well as their peak value. The maximum value of $C_{\text {ext }}$ of $0.3 \mu \mathrm{m}^{2}$ can be translated into $0.15 \lambda^{2}$ at the resonance wavelength $\lambda=1.4 \mu \mathrm{m}$. Alternatively, this result can be expressed as $C_{\text {ext }}=8 A_{\mathrm{SRR}}$ with the geometric area $A_{\mathrm{SRR}}=0.038 \mu \mathrm{m}^{2}$, which can be deduced directly from the electron micrographs (Fig. 1b).

The extinction cross-section is the sum of the absorption crosssection and the scattering cross-section, that is, $C_{\text {ext }}=C_{\text {abs }}+C_{\text {scatt }}$. To analyse theoretically the relative strengths of the two contributions, $C_{\text {abs }}$ and $C_{\text {scatt }}$, under the present conditions, we performed numerical calculations using the SRR geometrical parameters from the experiment and free-electron Drude model parameters from the literature for gold (see Methods). We included a glass substrate with a refractive index $n=1.5$ as a dielectric half-space. The curves in Fig. 3a show the corresponding results. The calculated absolute extinction crosssection (solid curve) agrees well with the measured one (filled circles in Fig. 3a), especially considering that no fitting has been attempted. About one-third of the peak extinction cross-section
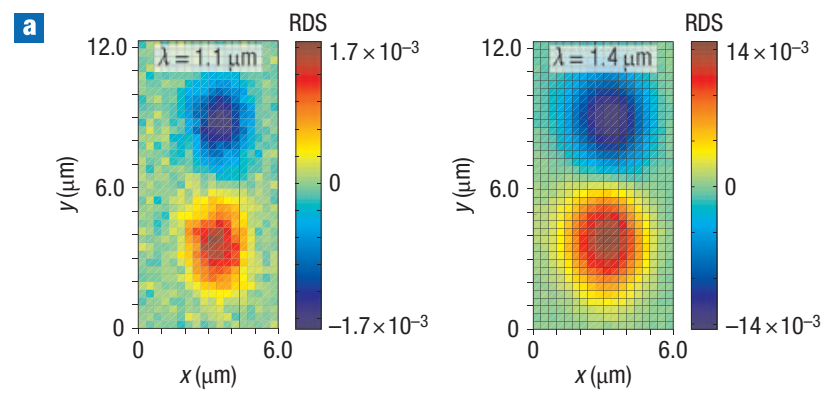

b

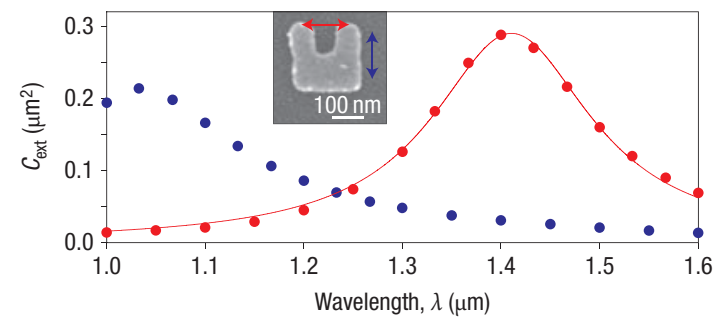

c

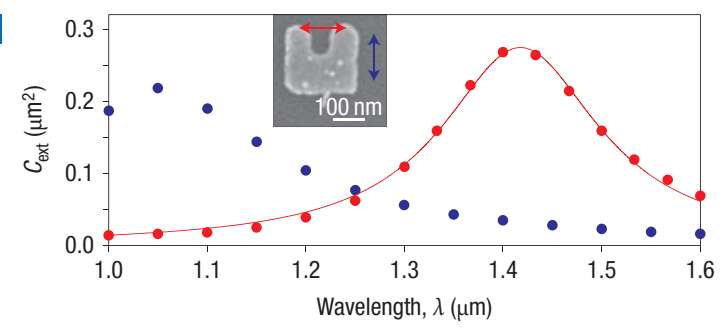

Figure 2 Measured absolute extinction cross-section spectra. a, Two typical sets of raw data of the measured RDS, one at resonance, the other in the tail of the resonance (compare red data and curves). $\mathbf{b}$, Measured absolute extinction cross-section spectrum $C_{\text {ext }}(\lambda)$ of an individual SRR. Two different polarization configurations are illustrated by the inset. c, As $\mathbf{b}$ for a different but similar SRR. The red solid curves are fits using a simple $L R C$ circuit model.

arises as a result of absorption (dashed curve) and two-thirds from scattering (dotted curve).

It is interesting to ask whether the extinction cross-section is a property of the SRR alone or whether it is influenced by its dielectric surrounding. Figure $3 \mathrm{~b}$ shows the extinction crosssection for the same SRR as in Fig. 3a, but within different homogeneous dielectric environments. It is obvious that all the curves in Fig. 3b differ from those in Fig. 3a, which means that the actual photon density of states of the half-space geometry significantly influences the coupling of the SRR to the light field. The resulting effects are as large as $30 \%$ in the peak extinction cross-section. In general, this result implies that comparing measured cross-sections of metallic nanoparticles on glass substrates (a very common geometry in experiments) with those of the calculated ones of such particles in a fictitious effective homogeneous medium is somewhat questionable.

Although these microscopic calculations agree well with the experimental results and have also given important additional insights, they do not really provide us with a simple intuitive understanding. Hence, we would like to connect the extinction cross-section of an individual SRR to the frequently used electric circuit mode ${ }^{5,6,16}$. In addition to the already mentioned capacitance $C$ and inductance $L$ of the SRR, an effective serial resistance $R$ is also considered. This resistance comprises an ohmic contribution 

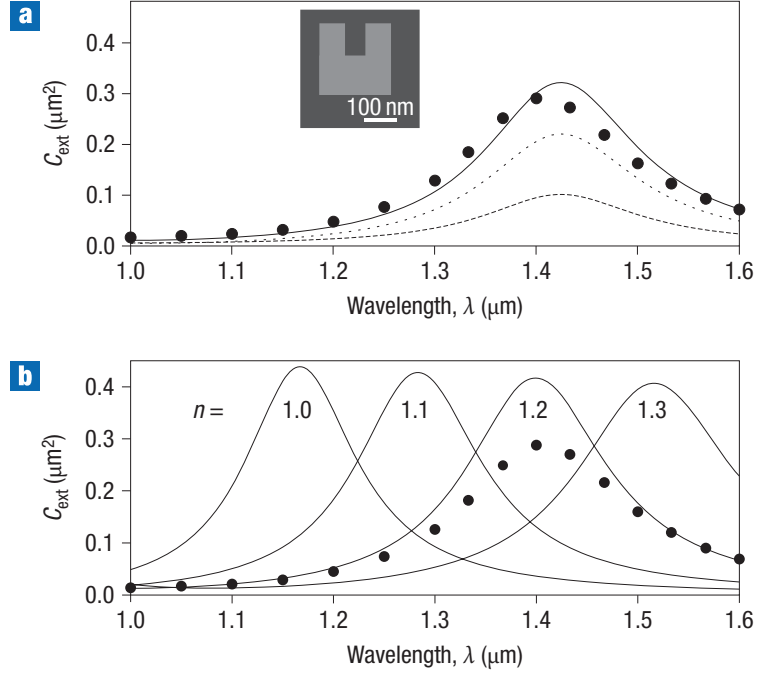

Figure 3 Calculated absolute cross-section spectra. a, The SRR is located on a glass substrate, and the incident linear polarization is horizontal. Shown are the extinction cross-section $C_{\text {ext }}$ (solid curve), the absorption cross-section (dashed curve) and the scattering cross-section (dotted curve) spectra.

b, $C_{\text {ext }}$ for the identical SRR, but embedded in a (fictitious) homogeneous medium with refractive index $n$ as indicated. The filled circles in $\mathbf{a}$ and $\mathbf{b}$ are the experimental data from Fig. $2 b$, reproduced here for comparison.

because of loss in the metal, $R_{\mathrm{Ohm}}$, as well as a contribution from the electromagnetic radiation, the radiation resistance $R_{\text {rad }}$. Kirchhoff's voltage law immediately allows the calculation of the current $I$ induced by the voltage drop Ed through the horizontally polarized incident electric field $E$ in the capacitor gap of width $d$. The power fraction $P_{\mathrm{R}} / P_{0}$ with the incident power $P_{0}$ and $P_{\mathrm{R}}=\left\langle I^{2} R\right\rangle \propto\left\langle E^{2} / R\right\rangle$ (where $\langle\cdot\rangle$ is the cycle average) immediately gives the cross-section $C_{\text {ext }}$ using the relation $P_{\mathrm{R}} / P_{0}=C_{\text {ext }} / A \leq 1$. For the resonance wavelength $\lambda_{\text {res }}$ and in the limit $C_{\text {ext }}\left(\lambda_{\text {res }}\right) \ll A$, it is simple to derive the expression $C_{\text {ext }}\left(\lambda_{\text {res }}\right)=\left(Z_{0} / R\right) d^{2}$ where $d^{2}$ is about oneninth of the geometric area of the SRR. Hence, the effective resistance $R$, which is much smaller than the vacuum impedance of $Z_{0}=\sqrt{ }\left(\mu_{0} / \varepsilon_{0}\right) \approx 376.7 \Omega$, leads to cross-sections that exceed the geometrical area. From the fit to the experimental data shown in Fig. $2 \mathrm{~b}$ and with $d=65 \mathrm{~nm}$ from the electron micrograph shown in Fig. 1b, we obtain an effective SRR resistance of $R=5.5 \Omega$ This value is reasonably close to the recent theoretical estimates of the SRR radiation resistance ${ }^{17}$ in a homogeneous dielectric environment. We can estimate $R_{\mathrm{rad}}=10 \Omega$ and $R_{\mathrm{Ohm}}=4 \Omega$ using the formulae given by Meyrath et al. ${ }^{17}$ and Busch et al. ${ }^{18}$, respectively. The relative magnitude of these values is qualitatively consistent with our above microscopic discussion on absorption and scattering. The dependence of $C_{\text {ext }}$ on frequency is clearly Lorentzian. Fitting this Lorentzian to the experimental data (see red solid curve in Fig. 2b) provides us with an effective SRR capacitance of $C=19 \mathrm{aF}$ and an effective SRR inductance of $L=30 \mathrm{fH}$.

In conclusion, we have measured the absolute extinction crosssection of individual magnetic SRRs to be about one-seventh of the square of the resonance wavelength at $\lambda=1.4 \mu \mathrm{m}$ using a modulation technique. Theoretical analysis shows that the absorption cross-section and the scattering cross-section contribute, respectively, one-third and two-thirds of that value. Theoretical analysis of the experiments further reveals that the relative influence of the substrate (half-space geometry) on the extinction cross-section of the metal nanoparticle at the interface can be as large as $30 \%$.
METHODS

EXPERIMENTAL

To obtain the effective Gaussian beam area $A=\pi / 2 r_{0}^{2}$ with the $1 / e^{2}$-intensity and Gaussian beam radius $r_{0}$, we have prepared specially tailored samples. In one run of standard electron-beam lithography and electron-beam evaporation, we fabricated, on a glass substrate (coated with a $5-\mathrm{nm}$ thin conductive layer of indium tin oxide), two orthogonal gold knife edges and the gold SRR of interest (see Fig. 1a,b). The thickness of the gold film was $25 \mathrm{~nm}$. By scanning the knife edges in the focal plane over the Gaussian focus by means of calibrated stepping motors and recording the error function, we directly determined $r_{0}$ and hence $A$. Importantly, recording data over a broad spectral range requires that the spectral behaviour $A(\lambda)$ is measured. In our experiments, $r_{0}$ linearly increased with wavelength $\lambda$ from $r_{0}(\lambda=1.0 \mu \mathrm{m})=1.2 \mu \mathrm{m}$ to $r_{0}(\lambda=1.6 \mu \mathrm{m})=2.1 \mu \mathrm{m}$, as expected for a diffraction-limited light spot. The light source in our experiments was a white-light laser (Fianium SC450, 2-W average power, 20-MHz repetition frequency), which was spectrally filtered by means of a grating monochromator (set to 1-nm resolution) and spatially filtered by means of a coupling into a single-mode optical fibre. The output of this fibre (below 100-nW average power) was imaged onto the sample using a $\times 20$ microscope lens (numerical aperture $=0.4$ ). An identical lens on the other side of the sample collected the light, which was sent on to a room-temperature germanium detector connected to a lock-in amplifier. Next, the SRR was moved to the region of the Gaussian focus and sinusoidally modulated by a piezoelectric transducer in one direction (here $y$-direction) at a frequency of $\sim 120 \mathrm{~Hz}$. For each wavelength, the RDS was recorded in the form of a complete image by scanning the sample in the $x y$-plane by means of a three-dimensional piezoelectric transducer stage (Fig. 2a). For all of the results actually depicted in this paper, the light impinged onto the SRR from the air side. We have also collected data for light impinging from the glass substrate side. Within experimental uncertainty, the measured extinction crosssection spectra are identical (although the spot sizes are different).

\section{THEORETICAL}

The numerical results depicted in this paper have been obtained by finitedifference time-domain calculations in three dimensions, using a home-made computer code. To avoid problems with staircasing, the SRR was modelled as a grid-aligned rectangular $U$ (see inset in Fig. 3a), the geometrical parameters of which were directly taken from the electron micrograph in Fig. 1b. The thickness of the gold film was $25 \mathrm{~nm}$. We described the dispersion of the gold dielectric function by the free-electron Drude model with parameters from the literature ${ }^{19}$, that is, plasma frequency $\omega_{\mathrm{pl}}=1.38 \times 10^{16} \mathrm{~s}^{-1}$ and collision frequency $\omega_{\text {coll }}=1.08 \times 10^{14} \mathrm{~s}^{-1}$. The system was discretized into uniform cells of size $\Delta x=\Delta y=2.5 \mathrm{~nm}$ and $\Delta z=1.25 \mathrm{~nm}$. To absorb the outgoing radiation, the computational domain was surrounded by a region of 10 cells of perfectly matched layers. The total system consisted of $192 \times 192 \times 264 \approx 10^{7}$ cells. For the calculation of the cross-sections, we injected a short pulse with a temporal Gaussian envelope and, hence, a broad frequency spectrum, through total-field/scattered-field boundaries ${ }^{20}$. During the simulation, an "on-the-fly" Fourier transform was performed to record the flux through two closed contours around the SRR. One of the contours lay inside the total-field region, and the other in the scattered-field region. Integration of the flux over the contours finally yielded the absorption and scattering cross-sections, respectively.

Received 20 February 2008; accepted 4 August 2008; published 7 September 2008.

References

1. Shalaev, V. M. Optical negative-index metamaterials. Nature Photon. 1, 41-48 (2007).

2. Soukoulis, C. M., Linden, S. \& Wegener, M. Negative refractive index at optical wavelengths. Science 315, 47-49 (2007)

3. Pendry, J. B., Schurig, D. \& Smith, D. R. Controlling electromagnetic fields. Science 312, $1780-1782(2006)$

4. Schurig, D. et al. Metamaterial electromagnetic cloak at microwave frequencies. Science 314, 977-980 (2006)

5. Pendry, J. B., Holden, A. J., Robbins, D. J. \& Stewart, W. J. Magnetism from conductors and enhanced nonlinear phenomena. IEEE Trans. Microwave Theory Tech. 47, 2075-2084 (1999).

6. Linden, S. et al. Magnetic response of metamaterials at 100 Terahertz. Science 306, 1351-1353 (2004)

7. Enkrich, C. et al. Magnetic metamaterials at telecommunication and visible frequencies. Phys. Rev. Lett. 95, 203901-1 (2005).

8. Arbouet, A. et al. Direct measurement of the single-metal-cluster optical absorption. Phys. Rev. Lett. 93, 127401-1 (2004).

9. Muskens, O. L. et al. Single-metal nanoparticle absorption spectroscopy and optical characterization. Appl. Phys. Lett. 88, 063109-1 (2006).

10. Shamonina, E. \& Solymar, L. Properties of magnetically coupled metamaterial elements. J. Magn. Magn. Mater. 300, 38-43 (2006).

11. Dolling, G., Wegener, M., Schädle, A., Burger, S. \& Linden, S. Observation of magnetization waves in negative-index photonic metamaterials. Appl. Phys. Lett. 89, 231118-231120 (2006). 
12. Liu, N. et al. Three-dimensional photonic metamaterials at optical frequencies. Nature Mater. 7, 31-37 (2008).

13. Sönnichsen, C., Franzl, T., Wilk, T., von Plessen, G. \& Feldmann, J. Drastic reduction of plasmon damping in gold nanorods. Phys. Rev. Lett. 88, 077402-1 (2002).

14. Bohren, C. F. \& Huffman, D. R. Absorption and Scattering of Light by Small Particles (Wiley, New York, 1983)

15. Rockstuhl, C. et al. On the reinterpretation of resonances in split-ring-resonators at normal incidence. Opt. Express 14, 8827-8836 (2006).

16. Engheta, N. Circuits with light at nanoscales: optical nanocircuits inspired by metamaterials. Science 317, 1698-1702 (2007).

17. Meyrath, T. P., Zentgraf, T. \& Giessen, H. Lorentz model for metamaterials: optical frequency resonance circuits. Phys. Rev. B 75, 205102-1 (2007).

18. Busch, K. et al. Periodic nanostructures for photonics. Phys. Rep. 444, 101-202 (2007).

19. Johnson, P. B. \& Christy, R. W. Optical constants of the noble metals. Phys. Rev. B 6 , 4370-4379 (1972).
20. Taflove, A. \& Hagness, S. C. Computational Electrodynamics: The Finite-Difference Time-Domain Method 3rd edn (Artech House, Boston, 2005).

Acknowledgements

We thank Costas M. Soukoulis for discussions. We acknowledge the support of the Deutsche Forschungsgemeinschaft (DFG) and the State of Baden-Württemberg through the DFG-Center for

Functional Nanostructures (CFN) within subprojects A1.2, A1.5, A5.2 and A5.3 as well as by the BMBFVerbund METAMAT and by the European Union project PHOME. The research of S.L. is also supported through a Helmholtz-Hochschul-Nachwuchsgruppe (VH-NG-232). The PhD research of N.F, M.K. and J.N. is further supported by the Karlsruhe School of Optics and Photonics (KSOP).

Author information

Reprints and permission information is available online at http://npg.nature.com/reprintsandpermissions/. Correspondence and requests for materials should be addressed to N.F. 